\title{
NONPLANAR LOOPS LEAVE THE VENEZIANO MODEL PHOTON MASSLESS
}

\author{
Omar FODA \\ Institute for Theoretical Physics, Princetonplein 5, P.O. Box 80.006, 3508 TA Utrecht, The Netherlands
}

Received 25 September 1986

\begin{abstract}
The absence of a pole at $p^{2}=0$ in the orientable nonplanar one-loop photon self-energy in the Veneziano model is verified. Thus the photon remains massless, and spontaneous symmetry breaking - at least as reported in this context in the literature - is not found.
\end{abstract}

A well-studied aspect of open-string perturbation theory [1] is that deforming a one-loop disk diagram with external vertices on each boundary (fig. la) leads to an orientable nonplanar configuration (fig. $1 \mathrm{~b}$ ) that is equivalent to a tree diagram with an intermediate closed string (fig. 1c). Thus, perturbative unitarity requires open strings to mix with closed strings via an open(reggeon)-closed(pomeron) vertex (fig. 2) (both terminologies will be used below interchangeably). The emergence of pomeron singularities in reggeon diagrams [2] is the subject of extensive literature, with many important results. For example, the condition that such mixed amplitudes factorize in accordance with unitarity gave the first indication that a string theory can be fully consistent only in a particular dimension [3].

What concerns us here is the remark that, subject to the constraints of kinematics and charge conservation, there exist direct transitions that mix open and closed string states, as can be realized by projecting the full vertex $V[a ; b, \tilde{b}]$ of fig. 2 on definite states $\langle a|V[a ; b, \tilde{b}]| b, \tilde{b}\rangle:$ fig. 3. [ $a$ and $b(\tilde{b})$ are the open and right(left)moving closed string oscillators, respectively.] Let us consider transitions between massless states. From the open string we have gauge bosons that may acquire a nonabelian charge through a Chan-Paton factor. However, since we are interested only in two-point functions with a single open-string vertex, following the rules and taking the trace over the single Chan-Paton factor gives an identically vanishing result; thus, only gauge singlets can contribute, and from now on we will talk about "the photon" having, of course, a generic neutral gauge boson in mind. On the other hand, if the pomeron belongs to the general case of an extended Virasoro-Shapiro model then it can contribute either of three massless states: a graviton, a dilaton, or an antisymmetric tensor [4].

In their analysis of the factorization of nonplanar diagrams [5], Cremmer and Scherk found that the photon $A_{\mu}$ could couple only to the antisymmetric tensor $B_{\mu \nu}$, both being odd under twist parity. Therefore, if the transition amplitude is indeed nonvanishing, one expects the model to reduce in the low-energy limit to a theory that contains both these fields together with a bilinear mixing term. But in that case the mass matrix would not be diagonal, $A_{\mu}$ and $B_{\mu \nu}$ would not be the observable physical fields, and one wonders what the physical spectrum is!

In ref. [6], the transition amplitude was not considered explicitly, but the non-planar one-loop contribution to the photon two-point function was computed, and found to contain a pole at $p^{2}=0$, where $p$ is the external momentum, signalling a direct transition between $A_{\mu}$ and the only candidate there is: $B_{\mu \nu}$. Then, to clarify the spectrum a four-dimensional field theory that supposedly has the salient features of the string effective field theory was proposed. It contains a photon, an antisymmetric tensor, and a mixing term:

$L=-\frac{1}{4} F_{\mu \nu} F^{\mu \nu}+\frac{1}{6} G_{\mu \nu \rho} G^{\mu \nu \rho}+\frac{1}{3} \lambda \epsilon_{\mu \nu \rho \sigma} A^{\mu} G^{\nu \rho \sigma}, \quad F_{\mu \nu}=\partial_{\mu} A_{\nu}-\partial_{\nu} A_{\mu}, \quad G_{\mu \nu \rho}=\partial_{\mu} B_{\nu \rho}+\partial_{\rho} B_{\mu \nu}+\partial_{\nu} B_{\rho \mu}$, 


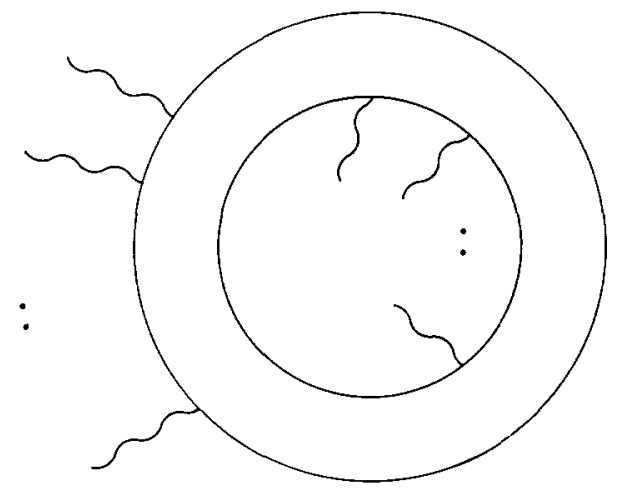

a

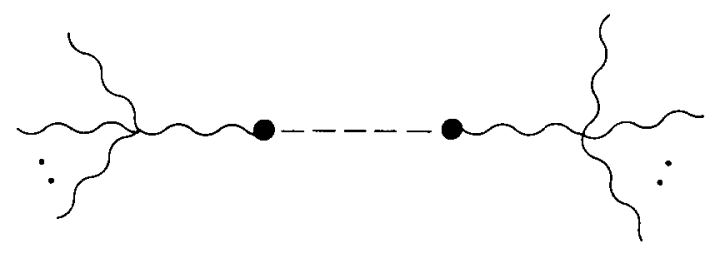

C

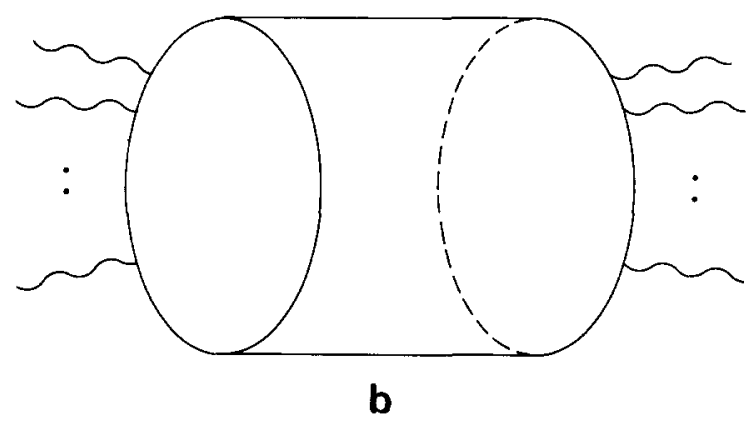

Fig. 1. (a) A one-loop disk diagram with external vertices on each boundary. (b) The nonplanar topology of the previous diagram is exhibited. (c) The equivalent tree diagram with an intermediate closed string.

and $\lambda$ is a coupling constant.

The reason (1) is a four-dimensional field theory, and could not be otherwise, is that the mixing term has to be invariant under the gauge transformations

$A_{\mu} \rightarrow A_{\mu}+\partial_{\mu} A, \quad B_{\mu \nu} \rightarrow B_{\mu \nu}+\partial_{\mu} A_{\nu}-\partial_{\nu} A_{\mu}$.

These are the field theory images of the world-sheet conformal invariances that should remain valid to decouple the negative norm states in a theory with both open and closed strings [7]. Recasting (1) in terms of diagonal fields, Cremmer and Scherk found that the photon combined with the single degree of freedom $B_{\mu \nu}$ has in $d=4$, and formed a massive vector boson. And since adding numerically the $(d-2)$ components of a massless $A_{\mu}$ in $d$ dimensions to the $\frac{1}{2}(d-2)(d-3)$ of a massless $B_{\mu \nu}$, one obtains $\frac{1}{2}(d-1)(d-2)$ : the correct number for a massive $B_{\mu \nu}$, one expects the symmetry breaking that showed up in (1) to obtain in arbitrary $d$, including $d=26$. However, there remains the puzzling fact that no suitable mixing term exists in $d \neq 4$. Given that the $A_{\mu}-B_{\mu \nu}$ mixing and the resulting symmetry breaking are frequently quoted in the string literature (see for example ref. $[8]$ ), it is an issue that requires clarification.

We need to evaluate the mixing term explicitly. The form of $V[a ; b, \tilde{b}]$ given in ref. [5] is rather intractable, but regarding the vertex as an open string tree amplitude with a reggeon on the boundary and a pomeron on the inside of the world sheet (fig. 4), it can be evaluated directly using the methods of Ademollo et al. [9]. We will

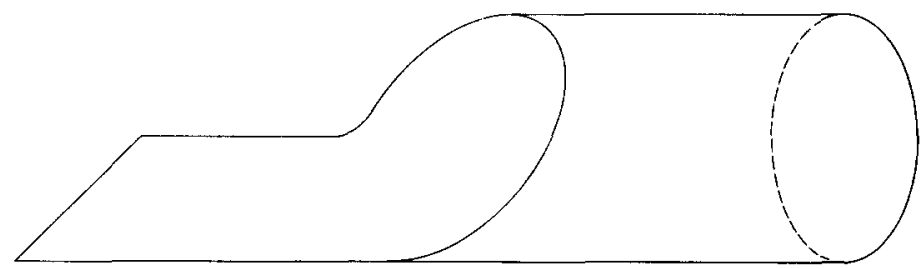

Fig. 2. The general open/closed string vertex. 


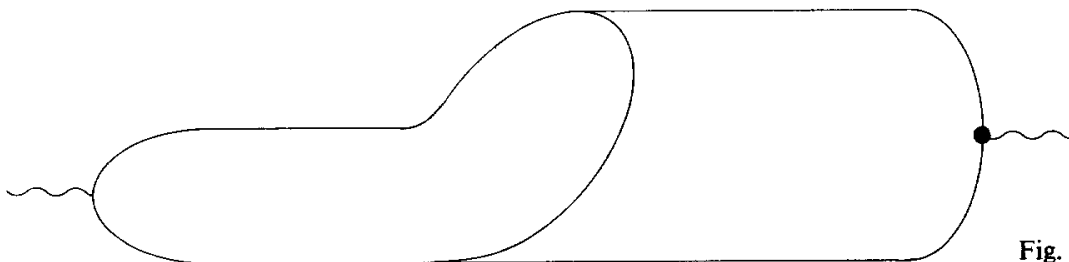

Fig. 3. The open/closed string state mixing term.

outline their derivation of the required result, and refer to them for further details. The tree amplitude for the scattering of $m$ pomerons $\mathbf{P}$ and $n$ reggeons $\mathbf{R}$ is

$A[m, n R]=\int \frac{\mathrm{d} V}{\mathrm{~d} V_{a b c}}\left\langle 0\left|\mathrm{~T}\left(\prod_{i=1}^{n} \prod_{j=1}^{m} V_{\alpha_{i}}\left(x_{i}, p_{i}\right) W_{\beta_{j} \beta_{j}}\left(\zeta_{j}, \bar{\zeta}_{j}, k_{j}\right)\right)\right| 0\right\rangle, \mathrm{d} V=\prod_{i=1}^{n} \prod_{j=1}^{m} \frac{\mathrm{d} x_{i}}{x_{1}} \frac{\mathrm{d}^{2} \zeta_{j}}{\left|\zeta_{j}\right|^{2}} \theta\left(x_{i+1}-x_{i}\right)$,

where dividing by $\mathrm{d} V_{a b c}$ stands for using the three-parameter projective invariance of the amplitude to fix any three real integration variables; $V_{\alpha_{i}}\left(x_{i}, p_{i}\right)\left[W_{\beta_{j} \tilde{\beta}_{j}}\left(\zeta_{j}, \bar{\zeta}_{j} ; k_{j}\right)\right]$ is a reggeon [pomeron] vertex of tensor indices $\alpha_{i}\left[\beta_{j}\right.$ and $\left.\widetilde{\beta}_{j}\right]$ momentum $p_{i}\left[k_{j}\right]$, located on the real line [complex plane] at $x_{i}=\exp \left(\tau_{i}\right)\left[\zeta_{i}=\exp \left(\tau_{j}+\mathrm{i} \sigma_{j}\right)\right]$. $T$ refers to ordering the pomerons according to the moduli of their variables $\zeta_{i}$, and with respect to the reggeons. The step function $\theta$ orders the latter on the real axis. Splitting $W_{\beta_{j} \bar{\beta}_{j}}\left(\zeta_{j}, \bar{\zeta}_{j} ; k_{j}\right)$ into its left- and right-moving "reggeon" components: $V_{\beta_{j}}\left(\zeta_{j}, \frac{1}{2} k_{j}\right) V_{\bar{\beta}_{j}}\left(\bar{\zeta}_{j}, \frac{1}{2} k_{j}\right)$, and applying the projective transformation

$\zeta \rightarrow \zeta^{\prime}=-(\zeta-\mathrm{i}) /(\zeta+\mathrm{i})$

to the string variables we map the real axis to the unit circle, the upper half-plane to the unit disk, and the pomeron variables $\zeta$ and $\bar{\zeta}$ are now related through an inversion $\zeta^{\prime}=1 / \bar{\zeta}^{\prime}$. Radial-ordering all vertices (the original reggeon vertices sit on the unit circle and have to be angular-ordered separately among themselves) we obtain the following expression:

$$
\begin{aligned}
& A[n \mathrm{R}, m \mathrm{P}]=\exp \left(\frac{1}{2} \mathrm{i} \pi k^{2}\right) \int_{\left|\zeta_{i}\right|<1} \prod_{i=2}^{m} \frac{\mathrm{d}^{2} \zeta_{i}}{\left|\zeta_{i}\right|^{2}} \int_{0}^{2 \pi} \prod_{j=2}^{n} \mathrm{~d} \phi_{j} \theta\left(\phi_{j}-\phi_{j-1}\right) \\
& \quad \times\left\langle-\frac{1}{2} k_{1}, \beta_{1}\left|\mathrm{~T}\left(\prod_{i=2}^{m} V_{\beta_{i}}\left(\zeta_{i}, \frac{1}{2} k_{i}\right)\right) V_{\alpha_{1}}\left(1, p_{1}\right) V_{\alpha_{j}}\left(\exp \left(\mathrm{i} \phi_{j}\right), p_{j}\right) \mathrm{T}\left(\prod_{i=2}^{m} V_{\tilde{\beta}_{i}}\left(1 / \zeta_{i}, \frac{1}{2} k_{i}\right)\right)\right| \frac{1}{2} k_{1}, \tilde{\beta}_{i}\right\rangle,
\end{aligned}
$$

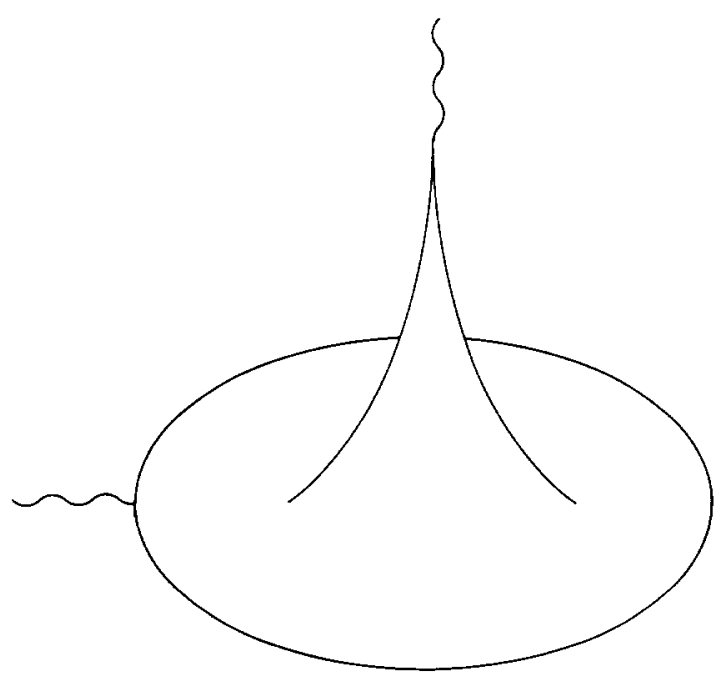

Fig. 4. The mixing term as an open string with one reggeon and one pomeron vertex. 
where $\exp \left(\mathrm{i} \phi_{j}\right) \equiv x_{j}, k=\sum_{i=1}^{m} k_{i}=-\sum_{j=1}^{n} P_{j}$, the phase factor follows from ordering the vertices, and projective invariance has been used to fix a reggeon vertex at $\phi_{1}=0$, and the two components of a pomeron vertex at $\zeta_{1} \rightarrow 0$, $1 / \zeta_{1} \rightarrow \infty$, which become the "reggeon" states at the end of the brackets.

(5) is in general divergent for excited pomerons due to possible reggeon-pomeron transitions and the occurrence of double poles, so it has to be handled with care; but this will pose no problem for the special case we are interested in. The point to notice about (5) is that radial-ordering splits the left- and right-moving components of each pomeron into separate reggeon vertices one to the left of all authentic reggeons and the other to the right, restricting (5) to a single pomeron and a single reggeon we arrive at the observation of Ademollo et al. [9] that the reggeon-pomeron vertex is a special case of the three-reggeon vertex [10,11] when all reggeons are on-shell and two of them carry the same momentum. Selecting the specific pomeron state we are interested in is achieved through considering suitable combinations of the polarization vectors of the component reggeons.

Consider now the photon-antisymmetric tensor vertex. This will follow from the collinear three-photon vertex through antisymmetrizing the polarization vectors of the two photons with the same momentum. But irrespectively of the polarization structure, the collinear three-photon vertex is identically vanishing as can be read off from the results of ref. [11], or directly by explicit computation

$A[1,2,3]=g\left[\eta_{1} \cdot k_{2} \eta_{2} \cdot \eta_{3}+\eta_{2} \cdot k_{3} \eta_{3} \cdot \eta_{1}+\eta_{3} \cdot k_{1} \eta_{1} \cdot \eta_{2}+\eta_{1} \cdot k_{2} \eta_{2} \cdot k_{3} \eta_{3} \cdot k_{1}\right]$,

where $\eta_{i}\left(k_{i}\right)$ is the polarization vector (external momentum) associated with the photon $i$. This is identically vanishing for physical, i.e. $\eta_{i} \cdot k_{i}=0$, collinear photons. Photons do interact in the Veneziano model (the lowenergy theory is not QED but a non-linear theory of the Born-field type [12]), but not collinearly!

This seems to explain the absence of a suitable mixing term: the amplitude for the required transition which has not been explicitly checked in ref. [6] is identically vanishing. However as mentioned above, a computation of the nonplanar photon self-energy was reported in ref. [6] to give a pole at $p^{2}=0$, with a finite positive residue. Thus we have to go through that computation in detail.

Computing loop diagrams with external states other than tachyons using oscillator methods is a straightforward exercise that can turn out to be extremely tedious. This is because, for practical reasons, one requires factorization of the various oscillator spaces, and this in general is spoilt by the tensor factors of the excitedstate vertices. (The situation for loops with four external bosonic vertices, due to the special properties of traces over fermionic zero mode $S_{0}$. All non-tachyonic loop computations reported in the literature are of this type [13] with the exception of ref. [14] which we discuss below.) A convenient trick to get around this problem can be gleaned from ref. [15] (see also section 4 of ref. [16]) and was independently suggested in ref. [17]: one should exponentiate the tensorial terms, so all vertices look tachyonic, perform all algebraic manipulations, then re-expand the exponentiated expressions, and pick up only those terms that would obtain from the original vertices. For example, consider the normal-ordered photon vertex, of momentum $k$ and polarization $\eta$ :

$V[\eta, k]=g \eta \cdot P(\tau): \exp [\mathrm{i} k \cdot X(\sigma=0, \tau)]:$

$X^{i}(\sigma=0, \tau)=q^{i}+p^{i} \tau+\mathrm{i}\left(\sum_{n=1}^{\infty}\left[\alpha_{n}^{i} \exp (-\mathrm{i} n \tau)-\alpha_{n}^{+i} \exp (\mathrm{i} n \tau)\right]\right), \quad P^{i}(\tau)=\frac{\partial}{\partial \tau} X^{i}(\sigma=0, \tau)$

$g$ is a dimensionless coupling constant, $\alpha_{n}^{+i}\left(\alpha_{n}^{i}\right)$ are the usual scaled creation (annihilation) operators and $\alpha^{\prime}$ has been set to $\frac{1}{2} . V[\eta, k]$ can be rewritten as

$V[\eta, k]=g \eta^{i} \frac{\partial}{\partial \zeta_{i}}: \exp (\mathrm{i} k \cdot X+\zeta \cdot P):\left.\right|_{\zeta^{i}=0}$,

and it is quite clear how to proceed.

Our task is to compute the nonplanar photon self-energy using (8). But this has already been done - together with the planar and non-orientable one-loop diagrams - by Jetzer and Lacki in ref. [14], and one can extract 
the desired result directly from their general expressions. Following ref. [14], with minor notational differences, the expression for the general non-planar loop diagram is

$$
\begin{aligned}
& A_{\mathrm{NP}}[1, \ldots, m]=\frac{(g / 2)^{m}}{\pi} \int_{0}^{1} \mathrm{~d} q \int_{0}^{1} \prod_{i=1}^{m-1} \theta\left(v_{i+1}-v_{i}\right) \mathrm{d} v_{i} f\left(q^{2}\right)^{-24} \frac{1}{q^{3}}\left(\frac{-2 \pi^{2}}{\ln q}\right)^{m} \\
& \times q^{-s / 4} \prod_{i<j}^{=}\left(\sin \left(\pi\left(v_{j}-v_{i}\right)\right) \prod_{n=1}^{\infty}\left[1-2 q^{2 n} \cos \left(2 \pi\left(v_{j}-v_{i}\right)\right)+q^{4 n}\right]\right)^{k_{i} \cdot k_{j}} \\
& \times \prod_{i<j}^{\neq}\left(\prod_{n=1}^{\infty}\left[1-2 q^{2 n-1} \cos \left(\pi\left(v_{j}-v_{i}\right)\right)+q^{4 n-2}\right]\right)^{k_{i} \cdot k_{j}} \\
& \times \prod_{i<j}\left(\exp \left\{\left(2 \pi^{2} / \ln q\right)\left[v_{i}+v_{j}-\frac{1}{2}\left(v_{i}^{2}+v_{j}^{2}\right)\right]\right\}\right)^{k_{i} \cdot k_{j}} \prod_{i<j} F\left(\tilde{B}_{i j}, \tilde{D}_{i j}\right)
\end{aligned}
$$

where $\alpha^{\prime}=\frac{1}{2}$, radial ordering of vertices through the step function $\theta$ is understood to apply to the vertices on each boundary separately, $\bar{\Pi}$ (夼) denotes taking the product over indices belonging to the same (different) boundary, the relation $\sum_{i<j} k_{i} k_{j}=0$ has already been used, and $s=\sum_{i=1}^{L} \sum_{j=L+1}^{m} k_{i} k_{j}$ is the invariant energy flowing through the closed string.

$$
\begin{aligned}
& \tilde{B}_{i j}\left(v_{j}-v_{i} ; q\right)=-\frac{1}{2}+\left(v_{j}-v_{i}\right)-\frac{\ln q}{2 \pi^{2}}\left(4 \pi \sum_{n=1}^{\infty} \frac{q^{n}}{1-q^{2 n}} \sin \left(2 \pi n\left(v_{j}-v_{i}\right)\right)\right), \\
& \tilde{D}_{i j}\left(v_{j}-v_{i} ; q\right)=\frac{\ln q}{2 \pi^{2}}-\left(\frac{\ln q}{2 \pi^{2}}\right)^{2}\left(4 \pi \sum_{n=1}^{\infty} \frac{q^{n}}{1-q^{2 n}} \cos \left(2 \pi n\left(v_{j}-v_{i}\right)\right) 2 \pi n\right), \\
& v_{m}=1
\end{aligned}
$$

and the rest of the notation is identical to that in ref. [16]. The function $F$ contains the polarization-dependent part of the amplitude, its general structure was described in ref. [14] and will be given below for the case we are interested in. Restricting (9) to a single vertex on each boundary we obtain

$$
\begin{aligned}
& A_{\mathrm{NP}}[1,2]=-\frac{(g / 2)^{2}}{\pi} \int_{0}^{1} \mathrm{~d} q \int_{1}^{1} \mathrm{~d} v f\left(q^{2}\right)^{-24} \frac{1}{q^{3}}\left(\frac{2 \pi^{2}}{\ln q}\right)^{2} q^{-s / 4}\left(\prod_{n=1}^{\infty}\left[1-2 q^{2 n-1} \cos (2 \pi v)+q^{4 n-2}\right]\right)^{k_{1} \cdot k_{2}} \\
& \quad \times\left\{\exp \left[\left(2 \pi^{2} / \ln q\right)\left(v+\frac{1}{2}-\frac{1}{2} v^{2}\right)\right]\right\}^{k_{1} \cdot k_{2}}\left\{\eta_{1} \cdot \eta_{2}\left[\left(\frac{\ln q}{2 \pi^{2}}\right)^{2} 4 \pi \sum_{n=1}^{\infty} \frac{q^{n}}{1-q^{2 n}} 2 n \pi \cos (2 n \pi v)\right]\right. \\
& \left.+k_{1} \cdot \eta_{2} k_{2} \cdot \eta_{1}\left[\frac{1}{2}-v+\frac{\ln q}{2 \pi^{2}}\left(4 \pi \sum_{n=1}^{\infty} \frac{q^{n}}{1-q^{2 n}} \sin (2 \pi n v)\right)\right]\left[\frac{1}{2}+v+\frac{\ln q}{2 \pi^{2}}\left(4 \pi \sum_{n=1}^{\infty} \frac{q^{n}}{1-q^{2 n}} \sin (2 \pi n v)\right)\right]\right\},
\end{aligned}
$$

now using $k_{1}=-k_{2}, k_{1} \cdot \eta_{2}=k_{2} \cdot \eta_{1}=0$, the second term in the large curly brackets vanishes identically, and taking the limit $s \sim k^{2} \rightarrow 0$, all terms raised to $k_{1} \cdot k_{2}$ contribute a factor of unity. Finally assuming a suitable infrared regulator to handle the divergence of the partition function in the limit $q \rightarrow 1$, it is clear that the whole expression is identically vanishing due to the $v$ integral over cosine functions.

Having obtained the same result in two basically different ways, and being in agreement with the absence of a suitable low-energy mixing term, we have to disagree with the conclusions of ref. [6]. It is sometimes argued 
that one can deduce the structure of excited-state amplitudes from that of their tachyonic analogues; see, for example, ref. [18]. And one reason we went in detail through the above computation was to show that the result is due to an integration over a function that belongs to the tensorial part of the amplitude.

The above analysis applies only to the massless states. Would mixing occur at higher levels? The easiest way to figure this would be to use the relationship between the reggeon-pomeron and the three-reggeon vertices discussed above. On the other hand, an interesting question is whether this form of spontaneous symmetry breaking can take place in fermionic string models. This is unlikely: the only consistent open fermionic string theory we know is the $\mathrm{SO}(32)$ open superstring. It contains no massless gauge singlets, thus mixing is prohibited through the Chan-Paton rules: only gauge singlets can travel through the closed string. What would happen upon gauge symmetry breaking and the possible appearance of a gauge singlet? There is actually an overriding reason for no mixing to occur. The open superstring mixes only with type-I closed strings ( that have $N=1$ rather than $N=2$ supersymmetry) [16], these contain no antisymmetric massless states, and it is unlikely that supersymmetry breaking can change the situation.

Finally, similar conclusions to those in ref. [6] were also reached in ref. [19]. There, a mixing term was written, that was valid in any dimension, but then the full invariance of the action under (2a) and (2b) was given up for a reduced invariance under a single combination of the two. This makes the connection of the obtained results to the original conformally-invariant string theory quite obscure. At any rate, our conclusions should apply here too, as they are independent of any effective field theory.

A. Abouelsaood brought ref. [6] to my attention; B. de Wit first noticed the absence of a suitable mixing term; P. Jetzer explained certain details of ref. [14] to me, and I discussed aspects of the problem with $\mathrm{H}$. Arodz. I wish to thank them all. This work was supported by Stichting voor Fundamenteel Onderzoek der Materie (FOM).

\section{References}

[1] M. Jacob, ed., Dual theory, Physics Reports reprint volume I (North-Holland, Amsterdam, 1974); J.H. Schwarz, ed., Superstrings (World Scientific, Singapore, 1985), and references therein.

[2] G. Frye and L. Susskind, Phys. Lett. B 31 (1970) 589; D.J. Gross, A. Neveu, J. Scherk and J.H. Schwarz, Phys. Rev. D 2 (1970) 697.

[3] C. Lovelace, Phys. lett. B 34 (1971) 500.

[4] A. D'Adda, R. D'Auria and S. Sciuto, Lett. Nuovo Cimento 5 (1972) 144.

[5] E. Cremmer and J. Scherk, Nucl. Phys. B 50 (1972) 222.

[6] E. Cremmer and J. Scherk, Nucl. Phys. B 72 (1974) 117.

[7] L. Brink, D. Olive and J. Scherk, Nucl. Phys. B 61 (1973) 173.

[8] J. Scherk, Rev. Mod. Phys. 47 (1975) 150; D. Clive, in: Dual theory, Physics Reports reprint volume I, ed. M. Jacob (North-Holland, Amsterdam, 1974) p. 142; J.H. Schwarz, Phys. Rep. 89 (1982) 254.

[9] M. Ademollo, A. D'Adda, R. D'Auria, E. Napolitano, P. DiVecchia, F. Gliozzi and S. Sciuto, Nucl. Phys. B 77 (1974) p. 189.

[10] S. Sciuto, Lett. Nuovo Cimento 2 (1969) 411; see also: P. DiVecchia, R. Nakayama, J.L. Petersen and S. Sciuto, Nordita preprint $86 / 8$; P. DiVecchia, Talk II Nobel Symp. (Marstrand, Sweden, June 1986), preprint Nordita-86/11.

[11] M: Ademollo, E. Del Giudice, P. DiVecchia and S. Fubini, Nuovo Cimento 19 (1974) 181.

[12] E. Fradkin and A.A. Tseytlin, Phys. Lett. B 136 (1985) 123.

[13] M.B. Green and J.H. Schwarz, Nucl. Phys. B 198 (1982) 441; D.J. Gross, J.A. Harvey, E. Martinec and R. Rohm, Nucl. Phys. B 267 (1986) 75

[14] P. Jetzer and J. Lacki, University of Geneva preprint UGVA-DPT 1986/06-508.

[15] M. Ademollo, A. D'Adda, R. D'Auria, F. Gliozzi, E. Napolitano, S. Sciuto and P. DiVecchia, Nucl. Phys. B 94 (1975) 221.

[16] J.H. Schwarz, Phys. Rep. 89 (1982) 223.

[17] H. Kawai, D.C. Lewellen and S.H.H. Tye, Nucl. Phys. B 269 (1986) 1.

[18] M.B. Green and J.H. Schwarz, Phys. Lett. B 151 (1985) 21.

[19] M. Kalb and P. Ramond, Phys. Rev. D 9 (1974) 2273. 